\title{
Dietary quality indices vary with sociodemographic variables and anthropometric status among Mexican adults: a cross-sectional study. Results from the 2006 National Health and Nutrition Survey
}

\author{
Xochitl Ponce, Sonia Rodríguez-Ramírez, Verónica Mundo-Rosas, Teresa Shamah, \\ Simón Barquera and Teresa González de Cossio* \\ Research Center on Nutrition and Health, National Institute of Public Health, Av. Universidad 655, \\ Col. Sta María Ahuacatitlán, Cuernavaca, Morelos, México CP 62508
}

Submitted 9 July 2012: Final revision received 28 June 2013: Accepted 25 July 2013: First published online 14 0ctober 2013

\begin{abstract}
Objective: To evaluate the dietary quality of Mexican adults' diet, we constructed three dietary quality indices: a cardioprotective index (CPI), a micronutrient adequacy index (MAI) and a dietary diversity index (DDI).

Design: Data were derived from the 2006 National Health and Nutrition Survey, which is a national survey representative of the Mexican population with a stratified, multistage, probabilistic sample design. Dietary intake was assessed from an FFQ with 101 different foods and daily nutrient intakes were computed. The CPI evaluated compliance with seven WHO recommendations for the prevention of CVD, the MAI evaluated the intake of six micronutrients based on the estimated average requirements from the US Institute of Medicine and the DDI was constructed based on the consumption of thirty different food groups.

Settings: Mexico.

Subjects: Mexican adults aged 19-59 years old.

Results: We evaluated the diet of 15675 males and females. Adjusted means and adjusted proportions by age and sex were computed to predict adherence to dietary recommendations. Rural inhabitants, those living in the South and those from the lowest socio-economic status reported a significantly higher CPI (4.5 (SE 0.08), $4 \cdot 3$ (SE $0 \cdot 08)$ and $4 \cdot 2$ (SE 0.09), respectively; $P<0 \cdot 05$ ), but a significantly lower MAI and DDI, compared with urban inhabitants, those from the North and those of upper socio-economic status $(P<0 \cdot 05)$.

Conclusions: The constructed diet quality indices identify nutrients and foods whose recommended intakes are not adequately consumed by the population. Given the epidemiological and nutritional transition that Mexico is experiencing, the CPI is the most relevant index and its components should be considered in Mexican dietary guidelines as well as in any food and nutrition programmes developed.
\end{abstract}

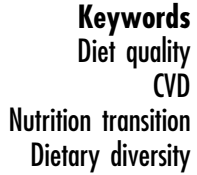

Keywords

CVD

Dietary diversity
An increase in overweight and obesity among the Mexican population was described by Rivera et $a l^{(1)}$. The National Health and Nutrition Survey carried out in 2006 (ENSANUT-2006) documented that $>70 \%$ of females and $67 \%$ of males aged $>20$ years were overweight or obese $\left(\geq 25 \cdot 0 \mathrm{~kg} / \mathrm{m}^{2}\right)^{(2,3)}$. Comparing previous National Nutrition Surveys, the prevalence of overweight and obesity increased from $34 \cdot 8 \%$ in 1988 to $61.0 \%$ in 1999 and to $69 \cdot 3 \%$ in $2006^{(4)}$. ENSANUT-2006 showed that $36 \cdot 8 \%$ of the adult Mexican population $(42 \cdot 2 \%$ of females and $30.3 \%$ of males) had metabolic syndrome according to the cut-off points of the National Cholesterol Education
Program Adult Treatment Panel III ${ }^{(2)}$. A concurrent increase in fat intake among the population in all regions of the country was reported as well as an increase in the purchase of refined sugars and carbohydrates ${ }^{(1)}$. However, this increase differed among regions and between urban and rural areas; fat, cholesterol and saturated fat intakes were higher in northern Mexico compared with the other regions as well as in urban localities compared with rural locations ${ }^{(5)}$. In addition, nutrient deficiencies still remain among the Mexican population $^{(4)}$. The Second Mexican National Nutrition Survey carried out in 1999 reported that the prevalence of 
Fe deficiency assessed by serum transferrin saturation among non-pregnant women aged 12-49 years was $40.5 \%$ and that $20 \%$ of the same population presented anaemia $^{(4)}$. Moreover, overlap of excess and deficiency in food intake exists. A prevalence of $6 \cdot 2 \%$ has previously been reported for maternal central adiposity along with childhood stunting in Mexico $^{(6)}$. This phenomenon reflects a process of nutritional transition with a polarization in different geographic areas and the coexistence of malnutrition in the country.

The relationship between dietary quality and health outcomes has been well documented in populations from 6 months to 99 years of age ${ }^{(7-9)}$. Food-based dietary guidelines have been developed to promote healthier eating patterns among the population ${ }^{(10)}$. Nutrient requirements and dietary recommendations as well as food guidelines are also useful approaches to assess dietary quality $^{(11,12)}$. Dietary indices based on nutrients ${ }^{(13)}$, foods ${ }^{(14)}$ and nutrients and foods ${ }^{(15)}$ have been developed to evaluate dietary quality. Dietary quality can be assessed by compliance to dietary guidelines such as those established by the WHO for the prevention of chronic diseases $^{(16,17)}$. Dietary quality indices have been used to evaluate and compare dietary quality at national ${ }^{(15)}$ and international levels ${ }^{(17)}$. Furthermore, dietary diversity and dietary variety have been used to reflect dietary quality because these measures are positively related to nutrient adequacy $^{(18,19)}$. Sociodemographic characteristics are also related to food consumption and probably to dietary quality as well ${ }^{(20)}$.

The alarming increase in excess weight among the adult Mexican population with its risk of CVD coexisting with micronutrient deficiencies evidences that diverse aspects of the Mexican diet have been modified during the last 20-30 years. Accordingly, an adequate dietary quality evaluation should focus on several dietary characteristics, not only on energy or micronutrient density $^{(21)}$. Such an evaluation would identify a healthrelated lack or surplus of food and nutrients. Those findings may be incorporated into current dietary guidelines. A shift from nutrient deficiency towards dietary excess has been observed.

Countries undergoing the nutrition transition present problems of dietary deficiency and excess; thus, it is not useful to examine only a single factor related to disease aetiology, but instead multiple risk factors that are sensitive to under- and overnutrition. In addition, dietary quality indices are a useful tool to quantify the risk of some health outcomes, biomarkers of diseases and risk of chronic diseases. Thus, our objectives in the present study were to observe the dietary quality of Mexican adults using indices sensitive to under- or overnutrition and to compare our results according to region, socio-economic status and BMI categories using three different indices: (i) a cardioprotective index (CPI); (ii) a micronutrient adequacy index (MAI); and (iii) a dietary diversity index (DDI).

\section{Materials and methods}

\section{Participants and study design}

For the present study we used information from ENSANUT$2006^{(3)}$, a survey that was conducted from October 2005 to May 2006 in 48304 households from the thirty-two states of Mexico. ENSANUT-2006 is a cross-sectional study with a multistage, probabilistic sample design. The survey is nationally representative because it includes all states within the country as well as urban and rural areas. The objective of the survey was to characterize the health and nutritional status of the Mexican population in all age groups. Methodological details of the survey may be found in a previous publication ${ }^{(3)}$. The analysis of the current paper includes information on a probabilistic sub-sample of one-third of the adult population (19-59 years old) in which dietary information was collected ( $n$ 16426). This sub-sample is also representative for the country, for urban and rural strata and the state level.

Informed consent was obtained from participating adults. The survey protocol was approved by the Ethics, Biosecurity and Research Committees of the National Institute of Public Health (NIPH), Mexico, the institute responsible for the survey.

\section{Data collection}

\section{Dietary information}

Dietary intake data were obtained using a $7 \mathrm{~d}$ semiquantitative FFQ. The FFQ includes 101 different foods and beverages classified into fourteen different groups. For each food, intake according to number of days in the week, times each day, portion size and number of portions consumed was queried during the $7 \mathrm{~d}$ prior to the interview $^{(22)}$. Nutrient intakes were computed using a food composition compilation developed by the $\mathrm{NIPH}^{(23)}$. Individuals with abnormal nutrient intake values were excluded from this analysis ( $n$ 543). A detailed description of dietary data collection can be found elsewhere ${ }^{(22)}$.

\section{Nutritional status}

Weight and height were measured by Lohman techniques $^{(24)}$. Field personnel were trained using the methodology proposed by Habicht ${ }^{(25)}$. Body weight was measured using a Tanita weight scale with a precision of $100 \mathrm{~g}$ that was calibrated daily. Height was measured using a stadiometer (Dynatop) with a precision of $1 \mathrm{~mm}$. BMI $\left(\mathrm{kg} / \mathrm{m}^{2}\right)$ was computed using weight and height measurements. We evaluated nutritional status using BMI cut-off points defined by WHO as follows: $\mathrm{BMI} \leq 24.9 \mathrm{~kg} / \mathrm{m}^{2}$ as normal, $\mathrm{BMI}=25 \cdot 0-29 \cdot 9 \mathrm{~kg} / \mathrm{m}^{2}$ as overweight and BMI $\geq 30 \cdot 0 \mathrm{~kg} / \mathrm{m}^{2}$ as obese ${ }^{(26)}$.

\section{Sociodemographic characteristics}

Region. The country was divided into four regions: (i) North (Baja California Norte and Sur, Coahuila, Chihuahua, 
Durango, Nuevo León, Sonora and Tamaulipas); (ii) Central (Aguascalientes, Colima, Estado de México, Guanajuato, Jalisco, Michoacán, Morelos, Nayarit, Querétaro, San Luis Potosí, Sinaloa and Zacatecas); (iii) metropolitan area of Mexico City; and (iv) South (Campeche, Chiapas, Guerrero, Hidalgo, Oaxaca, Puebla, Quintana Roo, Tabasco, Tlaxcala, Veracruz and Yucatán).

Residence area. A community with $<2500$ inhabitants was considered rural; otherwise it was classified as urban.

Socio-economic status. Information on household characteristics and possession of goods was used to construct an indicator of socio-economic status (SES) derived by the first component obtained through principal components analysis, which explained $46 \%$ of the variance. This indicator was validated in the previous Mexican Nutrition Survey ${ }^{(27)}$. The resulting standardized factor was divided into tertiles to categorize SES into low, middle and high groups.

\section{Dietary quality indices}

To evaluate dietary quality from the standpoint of compliance with dietary recommendations for the adult Mexican population, we used three indices of dietary quality which may point to the following health-related dietary dimensions: (i) potential for being cardioprotective, (ii) compliance with relevant micronutrients and (iii) diversity. For such analysis we developed pertinent dietary indices as follows.

\section{Cardioprotective index}

The CPI is based on seven WHO dietary guidelines for the prevention of $\mathrm{CVD}^{(16)}$. This index was used to evaluate the intake of a diet related to CVD. The CPI considers a recommended intake of protein $\geq 10 \%$ of total energy (\%TE), total fat $\leq 30 \% \mathrm{TE}$, SFA $\leq 10 \% \mathrm{TE}$, PUFA $<10 \% \mathrm{TE}$, cholesterol $<300 \mathrm{mg} / \mathrm{d}$, fibre $\geq 20 \mathrm{~g} / \mathrm{d}$, and a fruit and vegetable intake $\geq 400 \mathrm{~g} / \mathrm{d}$. For the construction of the index, 1 point was given if the individual complied with one of these dietary recommendations and 0 points otherwise. The maximum possible number of points was 7 and the minimum number was 0 ; the higher the CPI, the better the dietary quality from the standpoint of its cardioprotective effect.

\section{Micronutrient adequacy index}

This index was based on the Estimated Average Requirements from the US Institute of Medicine ${ }^{(28-30)}$. The index considers three minerals and three vitamins (Ca, Fe, Zn, folate, vitamin A and vitamin C) which were deficient in the population in the previous National Nutrition Survey $1999^{(4)}$. The MAI evaluated a recommended adequacy intake of $\mathrm{Ca} \geq 1000 \mathrm{mg} / \mathrm{d} ; \mathrm{Fe} \geq 6.0 \mathrm{mg} / \mathrm{d}$ for males and $\geq 8.1 \mathrm{mg} / \mathrm{d}$ for females; $\mathrm{Zn} \geq 9.4 \mathrm{mg} / \mathrm{d}$ for males and $\geq 6.8 \mathrm{mg} / \mathrm{d}$ for females; folate $\geq 320 \mu \mathrm{g} / \mathrm{d}$; vitamin A (retinol equivalents) $\geq 625 \mu \mathrm{g} / \mathrm{d}$ for males and $\geq 500 \mu \mathrm{g} / \mathrm{d}$ for females; and vitamin $\mathrm{C} \geq 75 \mathrm{mg} / \mathrm{d}$ for males and $\geq 60 \mathrm{mg} / \mathrm{d}$ for females. If the intake of the inidividual was at $100 \%$ or above for one of these nutrients, 1 point was given (for a maximum of 6 points and a minimum of 0 points). However, if the micronutrient intake was below the recommendation, no points were given. A higher MAI indicates a better dietary quality from the standpoint of its micronutrient adequacy.

\section{Dietary diversity index}

Food group classification. We classified the 101 foods included in the FFQ into thirty different food groups (Table 1). Food groups were created according to their micronutrient content and to the cultural aspects of the Mexican diet.

Dietary diversity. Dietary diversity was based on consumption of the thirty different food groups of $\geq 10 \mathrm{~g} / \mathrm{d}$ in the $7 \mathrm{~d}$ prior to the interview as recorded in the FFQ. Dietary diversity refers to the number of different food groups consumed, as reported in the FFQ used in the survey $^{(19)}$. Dietary diversity was divided into quintiles to construct the DDI. Individuals consuming seven different food groups or less scored 1 (DDI-1), those consuming eight to ten different food groups scored 2 (DDI-2), consumption of eleven or twelve different food groups scored 3 (DDI-3), consumption of thirteen to fifteen different food groups scored 4 (DDI-4) and, finally, individuals consuming sixteen different food groups or more scored 5 (DDI-5). The maximum score for the DDI was 5 points and the minimum was 1 point.

\section{Statistical analysis}

Dietary quality indices are presented by geographic region, area of residence, SES tertile and nutritional status. Descriptive statistics included means with their standard errors adjusted by age and sex, as well as the proportions adjusted by age and sex of the studied population who complied with dietary recommendations of each index.

Adjusted means for the three dietary indices as well as differences between population subgroups were calculated through linear regression models. We used Bonferroni $P$ adjustment for multiple comparisons when analysing differences within categories of each independent variable. Adjusted proportions of the population who complied with components of the dietary quality indices were calculated through logistic regression models and differences between regions, residence area, SES and BMI were analysed through the $\chi^{2}$ test. Individuals with missing information (anthropometric or SES characteristics) were excluded from the analysis. Pearson's correlation coefficient was used to test the association between the diet quality indices; $P<0.05$ was considered statistically significant, except when multiple comparisons were used.

The statistical software package Stata version 12 was used for statistical analyses. The SVY module in Stata was used to adjust analyses for the study design. 
Table 1 Food groups used for dietary diversity

\begin{tabular}{|c|c|}
\hline Food group & Food items \\
\hline 1. Tortilla & Soft, baked or fired tortillas \\
\hline 2. Non-fried corn products & Maize-based non-fried preparations such as tlacoyos, sopes, tacos \\
\hline 3. Fried foods and fried corn products & Tamales, fried tlacoyos, sopes, fried rice \\
\hline 4. Whole cereal products & Whole cereal bread, whole grains \\
\hline 5. Tubers and refined cereal products & White bread, refined grains, white bread, rice, pasta, refined wheat tortilla, atole \\
\hline 6. Sweetened breakfast cereals & Chocolate breakfast cereal, added-sugar breakfast cereal \\
\hline 7. Sweetened cereal- and sugar-based foods & Granola bar, amaranth bar \\
\hline 8. Green leafy vegetables & Cactus leaves, spinach and other dark green leaves \\
\hline 9. Other vegetables & Onion, cabbage, vegetables soup, tomato \\
\hline 10. Citrus fruits & Orange, tangerine, pineapple \\
\hline 11. Other fruit and natural fruit juices & Banana, pear, watermelon, natural unsweetened fruit juices \\
\hline 12. Poultry & Chicken, turkey \\
\hline 13. Eggs and egg-based stews & Eggs alone or in combination with ham, bacon \\
\hline 14. Meat and meat-based stews & Pork, beef, lamb, veal, meat balls \\
\hline 15. Processed meats & Ham, bacon, sausages, blood sausages \\
\hline 16. Fish and seafood & Fish (tuna, salmon, etc.), other seafood (shrimp, octopus, etc.) \\
\hline 17. Legumes & Beans, soyabeans, chick peas, lentils \\
\hline 18. Low-fat dairy & Skimmed milk, semi-skimmed milk, low-fat cheese, low-fat yoghurt \\
\hline 19. Full-fat dairy & Whole milk, high-fat cheese, high-fat yoghurt \\
\hline 20. Sweetened milk & Milkshake, chocolate milk, sugared milk with coffee or other flavours \\
\hline 21. Fortified milk and foods & Liconsa milk \\
\hline 22. Milk- and egg-based desserts & Crème caramel, petite Suisse \\
\hline 23. Vegetable oils and seeds & Margarine, peanuts, nuts, almonds, avocado, maize oil, olive oil \\
\hline 24. Animal fats & Cream, butter \\
\hline 25. Sweets & Sugar, sweets, chocolate, marmalades, jellies \\
\hline 26. Sweetened beverages and sodas & Sweetened soft drinks, sweetened fruit beverages, sweetened fruit juices \\
\hline 27. Bakery & Muffins, biscuits, cakes, pies, sponge cake, doughnuts \\
\hline 28. Fast foods & Pizza, hot dogs, hamburgers, sandwich \\
\hline 29. Salted high energy-dense foods & Potato chips, maize chips and similar cereal-based fried and salted snacks \\
\hline 30. Alcoholic beverages & Tequila, beer \\
\hline
\end{tabular}

\section{Results}

\section{Study sample characteristics}

We analysed data for 16494 adults aged between 19 and 59 years old. We excluded 205 pregnant or lactating women and 543 individuals with missing dietary information or implausible dietary intake data (because they presented intakes and adequacy percentages more than $5 \mathrm{sD}$ above the mean ( $n$ 164) or energy intake $<25 \%$ of the mean ( $n$ 379)). We also excluded individuals without socio-economic ( $n$ 55) or anthropometric ( $n$ 16) information. The final sample size for analyses was 15675 adults, representing 47313935 adults at the national level. We did not observe statistical differences for age and sex variables between the final study sample and the exclusion sample.

There were more females than males in the studied sample $(59 \cdot 8 \% \quad v \cdot 40 \cdot 2 \%$, respectively); $24 \cdot 5 \%$ of the sample was 19-29 years old, 33.8\% was 30-39 years old, $25 \cdot 7 \%$ was $40-49$ years old and $16 \cdot 1 \%$ of the sample was 50-59 years old (data not shown). Table 2 summarizes sociodemographic and anthropometric characteristics of the studied population.

\section{Dietary quality indices}

Calculated indices are presented in Table 3. Results showed that the CPI was significantly higher among rural participants than urban participants $(4.5$ (SE 0.09) $v$. 4.3 (SE 0.09 ); $P<0 \cdot 05$ ). Participants living in southern
Table 2 General characteristics of the population*: Mexican adults aged 19-59 years, 2006 National Health and Nutrition Survey (ENSANUT-2006)

\begin{tabular}{|c|c|c|}
\hline Characteristic & $\begin{array}{c}\text { Sample size } \\
(n)\end{array}$ & $\begin{array}{l}\% \text { of total } \\
\text { population }\end{array}$ \\
\hline \multicolumn{3}{|l|}{ Area of residence } \\
\hline Rural & 6452 & $19 \cdot 5$ \\
\hline Urban & 9223 & $80 \cdot 5$ \\
\hline \multicolumn{3}{|l|}{ Region of the country } \\
\hline North & 2913 & $19 \cdot 9$ \\
\hline Centre & 5929 & $29 \cdot 7$ \\
\hline Mexico City & 667 & $20 \cdot 5$ \\
\hline South & 6166 & $29 \cdot 9$ \\
\hline \multicolumn{3}{|l|}{ Socio-economic status (SES) } \\
\hline Low SES & 7673 & $30 \cdot 6$ \\
\hline Middle SES & 5048 & $34 \cdot 5$ \\
\hline High SES & 2954 & $34 \cdot 8$ \\
\hline \multicolumn{3}{|l|}{ Nutritional status } \\
\hline Normal weight $\left(\mathrm{BMI} \leq 24.9 \mathrm{~kg} / \mathrm{m}^{2}\right)$ & 5181 & $32 \cdot 7$ \\
\hline Overweight $\left(\mathrm{BMI}=25 \cdot 0-30 \cdot 0 \mathrm{~kg} / \mathrm{m}^{2}\right)$ & 5814 & $37 \cdot 3$ \\
\hline Obese $\left(\mathrm{BMI} \geq 30 \cdot 0 \mathrm{~kg} / \mathrm{m}^{2}\right)$ & 4680 & $29 \cdot 9$ \\
\hline
\end{tabular}

Mexico presented a significantly higher CPI $(4 \cdot 7$ (SE 0 066)) compared with participants in the other three regions $(P<0 \cdot 05)$. The CPI significantly and progressively decreased $(P<0 \cdot 05)$ from low SES $(4.5$ (SE $0 \cdot 08)$ ) to middle SES $(4 \cdot 3(\operatorname{SE} 0 \cdot 08))$ to high SES $(4 \cdot 2$ (SE 0.09)).

Urban participants reported a significantly higher MAI compared with their rural counterparts (1.9 (SE 0.08) $v$. 1.7 (se $0 \cdot 10) ; P<0 \cdot 05$ ). Participants from central Mexico 
Table 3 Mean dietary quality index scores of the population* according to area of residence, geographic region and socio-economic status: Mexican adults aged 19-59 years, 2006 National Health and Nutrition Survey (ENSANUT-2006)

\begin{tabular}{|c|c|c|c|c|c|c|}
\hline \multirow[b]{3}{*}{ Characteristic } & \multirow{2}{*}{\multicolumn{2}{|c|}{$\begin{array}{c}\text { Cardioprotective index (CPI) } \\
\text { Minimum score: } 0 \\
\text { Maximum score: } 7\end{array}$}} & \multirow{2}{*}{\multicolumn{2}{|c|}{$\begin{array}{c}\text { Micronutrient adequacy index (MAI) } \\
\text { Minimum score: } 0 \\
\text { Maximum score: } 6\end{array}$}} & \multirow{2}{*}{\multicolumn{2}{|c|}{$\begin{array}{c}\text { Dietary diversity (DD) } \\
\text { Minimum score: } 1 \\
\text { Maximum score: } 30\end{array}$}} \\
\hline & & & & & & \\
\hline & Mean & SE & Mean & SE & Mean & $\mathrm{SE}$ \\
\hline Total ( $n 15675)$ & $4 \cdot 4$ & 0.08 & 1.9 & 0.08 & $13 \cdot 6$ & $0 \cdot 17$ \\
\hline \multicolumn{7}{|l|}{ Area } \\
\hline Rural (n 6452) & $4 \cdot 5^{a}$ & 0.09 & $1 \cdot 7^{\mathrm{a}}$ & $0 \cdot 10$ & $12 \cdot 2^{a}$ & 0.19 \\
\hline Urban ( $n$ 9223) & $4 \cdot 3^{\mathrm{b}}$ & 0.09 & $1 \cdot 9^{b}$ & 0.08 & $14 \cdot 0^{\mathrm{b}}$ & $0 \cdot 17$ \\
\hline \multicolumn{7}{|l|}{ Region of the country } \\
\hline North $(n$ 2913) & $4 \cdot 1^{a}$ & 0.06 & $1 \cdot 8^{a}$ & 0.08 & $13 \cdot 2^{a}$ & 0.18 \\
\hline Center ( $n$ 5929) & $4 \cdot 5^{\mathrm{b}}$ & 0.06 & $1.9^{\mathrm{b}}$ & 0.09 & $13 \cdot 5^{a}$ & 0.19 \\
\hline Mexico City ( $n$ 667) & $4 \cdot 6^{\mathrm{b}, \mathrm{c}}$ & 0.09 & $1 \cdot 9^{b}$ & $0 \cdot 12$ & $14 \cdot 9^{b}$ & 0.23 \\
\hline South ( $n$ 6166) & $4 \cdot 7^{c}$ & 0.06 & $1 \cdot 8^{a}$ & 0.89 & $13 \cdot 1^{\mathrm{c}}$ & 0.19 \\
\hline \multicolumn{7}{|c|}{ Socio-economic status (SES) } \\
\hline Low SES $(n 7673)$ & $4 \cdot 5^{a}$ & 0.08 & $1 \cdot 7^{\mathrm{a}}$ & 0.08 & $12 \cdot 6^{a}$ & 0.19 \\
\hline Middle SES ( $n$ 5048) & $4 \cdot 3^{b}$ & 0.08 & $1 \cdot 9^{b}$ & 0.09 & $13 \cdot 9^{b}$ & $0 \cdot 18$ \\
\hline High SES (n 2954) & $4 \cdot 2^{b, c}$ & 0.09 & $2 \cdot 2^{c}$ & 0.09 & $14 \cdot 9^{\mathrm{C}}$ & 0.19 \\
\hline
\end{tabular}

Values are means (with their standard errors) adjusted by age and sex through linear regression models.

${ }^{*}$ Total sample size: 15675 , weighted cases: 47313935 Mexican adults.

a,b,c Mean values within categories of each independent variable within a column with unlike superscript letters were significantly different using Bonferroni adjustment $(P<0.05$ for area, $P<0.012$ for region, $P<0.016$ for SES.

(1.9 (se 0.09)) and metropolitan Mexico City (1.9 (SE 0.12)) reported a significantly higher MAI $(P<0 \cdot 05)$ than those from northern Mexico (1.8 (SE 0.08)) or southern Mexico $(1 \cdot 8(\mathrm{SE} 0 \cdot 89))$. The MAI significantly and progressively increased $(P<0.05)$ with SES from the low SES $(1.7$ (SE 0.08)) to the middle (1.9 (SE 0.09)) and the high SES tertile $(2 \cdot 2($ se $0 \cdot 09))$.

Urban participants reported a significantly higher DDI than participants living in rural areas $(14 \cdot 0(\operatorname{SE} 0 \cdot 17) v$. $12 \cdot 2$ (SE $0 \cdot 19$ ); $P<0 \cdot 05$ ). Those living in Mexico City reported a more diversified diet $(14.9(\mathrm{SE} 0 \cdot 23))$ than those living in the other three regions $(P<0 \cdot 05)$. The DDI significantly $(P<0.05)$ and progressively increased from the low $(12 \cdot 6(\operatorname{se} 0 \cdot 19))$ to the middle $(13 \cdot 9(\operatorname{se~} 0 \cdot 18))$ and the high $(14 \cdot 9$ (sE 0.19)) SES category.

Daily compliance to components of the CPI is described in Fig. 1. Almost all components of the CPI were significantly better in rural than in urban participants, except for the recommended fruit and vegetable intake, which was significantly higher in urban than in rural adults (35.6\% v. 25.5\%; $P<0 \cdot 05$ ). The proportion of participants who attained the recommended intake was significantly better in rural areas than in urban ones with regard to total fat $(81 \cdot 2 \% v$. 65.1\%; $P<0 \cdot 05)$, SFA $(82 \cdot 9 \% \quad v .72 \cdot 4 \%$; $P<0.05)$, cholesterol (81.9\% v. 75.6\%; $P<0.05)$ and fibre $(59 \cdot 5 \%$ v. $50 \cdot 7 \% ; P<0 \cdot 05)$.

Protein $(80 \cdot 5 \%)$, total fat $(57 \cdot 1 \%)$, SFA $(68 \cdot 1 \%)$, as well as the recommended intakes for cholesterol $(72 \cdot 0 \%)$ and fruits and vegetables (26.3\%), demonstrated significantly lower compliance of participants living in northern Mexico compared with those living in the other regions of the country $(P<0 \cdot 05)$. Participants from northern Mexico complied significantly better with the recommended intake of PUFA $(93.8 \%)$ than those from other regions of the country. The proportion of participants who complied with the recommended intake of fruits and vegetables significantly and progressively decreased from the high to the middle to the low SES category $(42 \cdot 7 \%, 32 \cdot 3 \%$ and $24.9 \%$, respectively; $P<0.05)$. The same was observed with dietary intake of protein $(85.5 \%, 82.5 \%$ and $81.2 \%$; $P<0 \cdot 05)$. The proportion of participants who complied with the recommended intake of total fat $(58 \cdot 8 \%, 68 \cdot 9 \%$ and $77 \cdot 9 \%)$, fibre $(49 \cdot 5 \%, 52 \cdot 2 \%$ and $56 \cdot 2 \%)$ and SFA $(66 \cdot 3 \%, 75 \cdot 4 \%$ and $82.5 \%)$, as well as the recommended intake for cholesterol $(74 \cdot 3 \%, 75 \cdot 9 \%$ and $80 \cdot 7 \%)$, significantly and progressively increased from the high to the middle to the low SES category $(P<0 \cdot 05)$. There was no significant difference between the proportion of participants who complied with the cardioprotective indicators according to nutritional status except for the recommended intake of fibre, which was significantly higher among overweight compared with obese participants $(54 \cdot 2 \%$ v. 50.4\%; $P<0 \cdot 05)$.

Daily compliance to components of the MAI is summarized in Fig. 2. The proportion of participants living in urban areas, as compared with those living in rural areas, meeting the recommended intake of various micronutrients was significantly higher for: $\mathrm{Zn}(46 \cdot 2 \% \mathrm{v}$. $38.6 \% ; P<0.05)$, folate $(23.3 \% v$. $19.5 \% ; P<0.05)$, vitamin $\mathrm{A}(13.0 \% \quad v .8 \cdot 1 \% ; P<0.05)$ and vitamin $\mathrm{C}$ $(19.9 v .14 .8 \% ; P<0.05)$. There was a significantly $(P<0.05)$ lower proportion of participants living in the North as compared with other regions of the country who complied with the recommended intake of Ca. The proportion of participants who complied with the recommended folate intake was significantly lower for those living in the South $(19.1 \% ; P<0.05)$ as compared with the North (25.9\%), central Mexico (24.8\%) and 

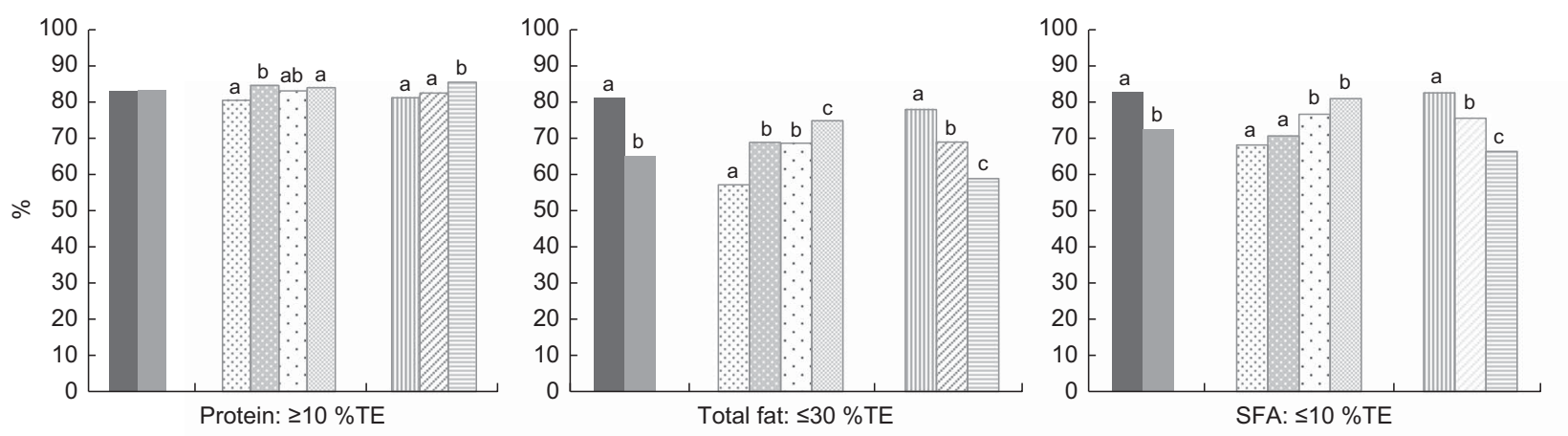

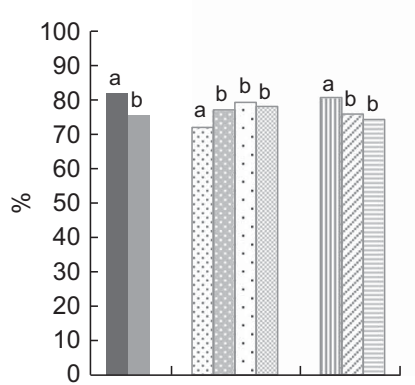

TC: $<300 \mathrm{mg}$

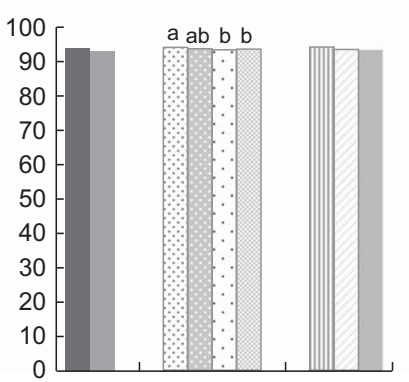

PUFA: $<10 \%$ TE

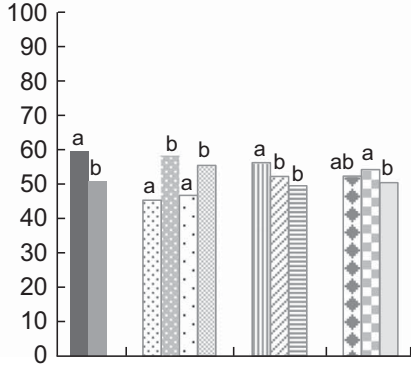

Fibre: $\geq 20 \mathrm{~g}$

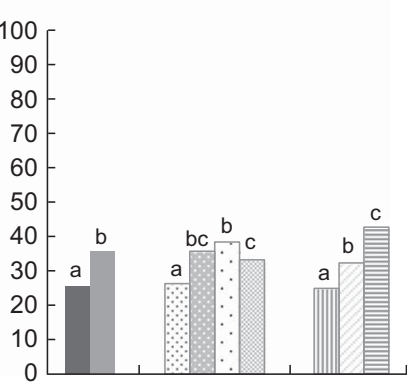

$\mathrm{F} \& \mathrm{~V}: \geq 400 \mathrm{~g}$

Fig. 1 Daily compliance with components of the cardioprotective index (CPI) according to area of residence ( $\square$, rural, $n$ 6452; $\square$, urban, $n$ 9223), geographic region (- North, $n$ 2913; $\square$, Centre, $n$ 5929; $\square$, Mexico City, $n 667$; $\square$, South, $n$ 6166), socioeconomic status (SES) tertile (四, low SES, $n$ 7673; $\square$, middle SES, $n$ 5048; 目, high SES, $n$ 2954) and nutritional status (疄, normal weight, $n$ 5181; $\square$, overweight, $n$ 5814; $\square$, obese, $n$ 4680) among Mexican adults aged 19-59 years, 2006 National Health and Nutrition Survey (ENSANUT-2006). Values are proportions adjusted by age and sex through logistic regression models. ${ }^{\mathrm{a}, \mathrm{b}, \mathrm{c}}$ Adjusted proportions within categories of each independent variable with unlike superscript letters were significantly different $(P<0 \cdot 05)$. WHO dietary guidelines for the prevention of $C D^{(16)}$ are indicated below each plot $(\% \mathrm{TE}$, percentage of total energy; $\mathrm{TC}$, total cholesterol; F\&V, fruit and vegetables)

Mexico City (20.9\%). The proportion of participants living in Mexico City who complied with the recommended intake of vitamin C $(23.5 \%)$ was significantly higher $(P<0 \cdot 05)$ than the proportions of compliers living in other regions of the country. A significantly higher proportion of participants in the high SES category complied with the recommended intake of $\mathrm{Ca}(38.5 \%)$ than participants from the middle $(31 \cdot 2 \%)$ or low SES $(31 \cdot 4 \%)$ category $(P<0 \cdot 05)$. The proportion of participants who attained the recommended intake of $\mathrm{Zn}(38 \cdot 0 \%, 43 \cdot 2 \%$ and $52 \cdot 1 \%)$, vitamin $\mathrm{A}(7 \cdot 6 \%, 10 \cdot 9 \%$ and $17 \cdot 1 \%)$, Fe $(74.7 \%, 75 \cdot 5 \%$ and $78 \cdot 8 \%)$, folate $(19 \cdot 7 \%, 23 \cdot 7 \%$ and $23.9 \%)$ and vitamin $\mathrm{C}(13.9 \%, 19 \cdot 3 \%$ and $23 \%)$ significantly and progressively increased from low to middle to high SES $(P<0 \cdot 05)$. A significantly lower proportion of obese participants complied with the recommended intake of $\mathrm{Ca}(29 \cdot 4 \%)$ compared with normal-weight $(34 \cdot 8 \%)$ or overweight participants $(36.5 \% ; P<0.05)$. There was significantly better compliance with the recommended intakes of vitamin $\mathrm{A}$ and vitamin $\mathrm{C}$ by those who were obese $(13.9 \%$ and $20.9 \%$, respectively) than by normalweight or overweight participants $(P<0 \cdot 05)$.

Figure 3 describes the proportion of participants in each quintile of the DDI. The proportion who reported a low DDI was significantly higher in rural than in urban participants (DDI-1: $5 \cdot 8 \% v \cdot 2 \cdot 1 \%$, DDI-2: \% $23 \cdot 4 v \cdot 11 \cdot 5 \%$, DDI-3: $23.5 \% \quad v \cdot 17 \cdot 6 \% ; P<0.05)$. The proportion of participants who consumed seven different foods groups or fewer (DDI-1) was significantly higher in those living in southern Mexico (4.4\%) compared with the other three regions of the country $(P<0 \cdot 05)$. The proportion with a high DDI was significantly higher among those living in Mexico City (DDI-5: 44.9\%) compared with other regions of the country $(P<0 \cdot 05)$. The proportion of participants reporting a low DDI was significantly higher in the low SES (DDI-1: $5.9 \%$, DDI-3: 23.6\%) compared with the other SES categories $(P<0 \cdot 05)$. The proportion of participants with a high DDI was significantly higher $(P<0 \cdot 05)$ among those of high SES (DDI-5: 42.5\%) compared with those of low SES (DDI-5: 17.7\%) or middle SES (DDI-5: 28.8\%).

Correlation coefficients between the indices were as follows: DDI with MAI $(r=0 \cdot 435, P<0 \cdot 001)$; DDI with CPI $(r=0 \cdot 106, P<0 \cdot 001)$; and CPI with MAI $(r=0 \cdot 258$, $P<0 \cdot 001)$.

\section{Discussion}

In the present study we evaluated three dietary quality indices among the adult Mexican population according to 

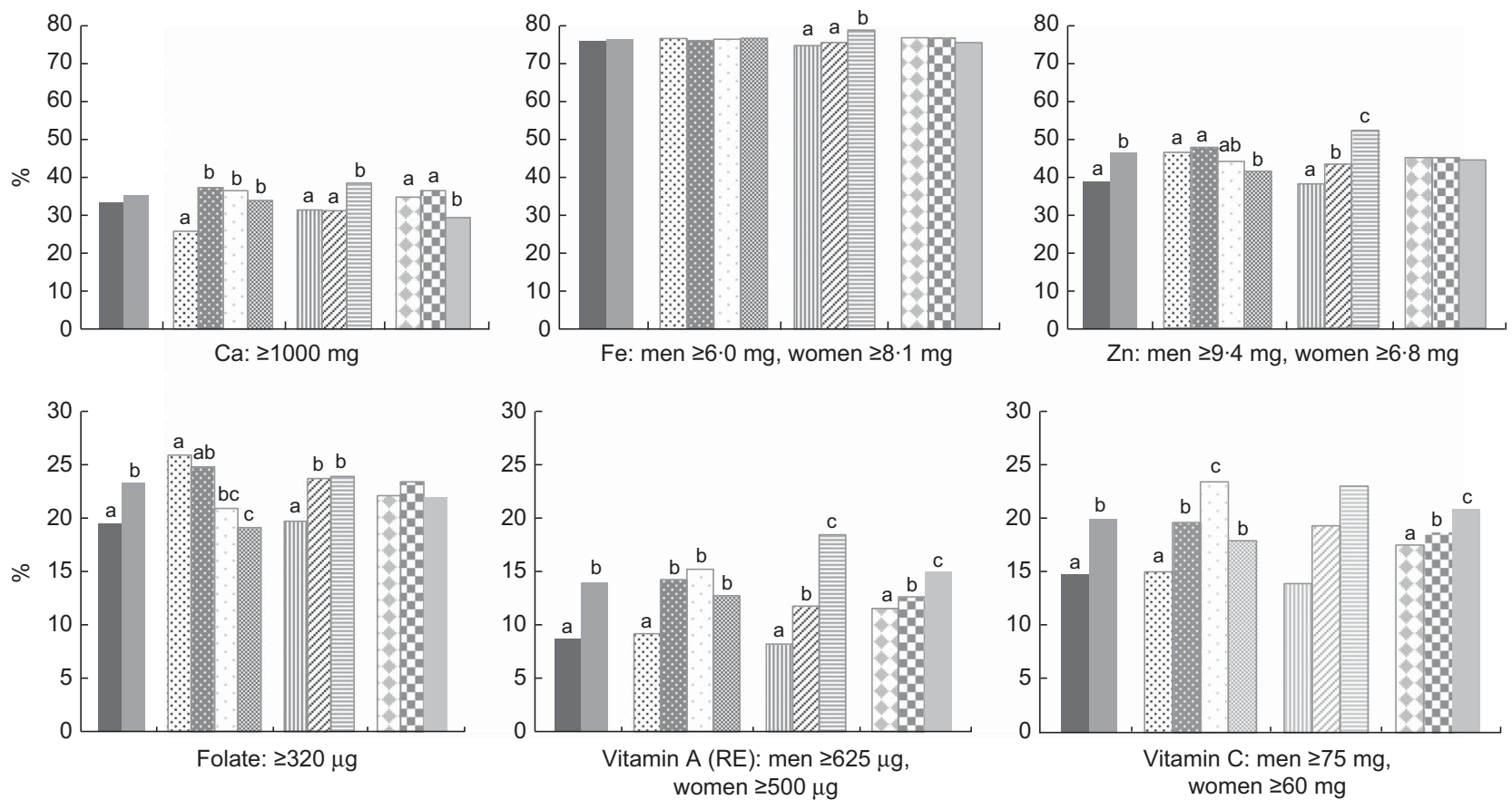

Fig. 2 Daily compliance with components of the micronutrient adequacy index (MAl) according to area of residence ( $\square$, rural, $n$ 6452; $\square$, urban, $n$ 9223), geographic region ( $\square$, North, $n$ 2913; $\square$, Centre, $n$ 5929; $\square$, Mexico City, $n$ 667; $\square$, South, $n$ 6166), socio-economic status (SES) tertile (四, low SES, $n$ 7673; $\square$, middle SES, $n$ 5048; 貝, high SES, $n$ 2954) and nutritional status (圈, normal weight, $n$ 5181; $\square$, overweight, $n$ 5814; $\square$, obese, $n$ 4680) among Mexican adults aged 19-59 years, 2006 National Health and Nutrition Survey (ENSANUT-2006). Values are proportions adjusted by age and sex through logistic regression models. ${ }^{\mathrm{a}, \mathrm{b}, \mathrm{c}}$ Adjusted proportions within categories of each independent variable with unlike superscript letters were significantly different $(P<0 \cdot 05)$. Recommended micronutrient intakes ${ }^{(28-30)}$ are indicated below each plot (RE, retinol equivalents)

sociodemographic and nutritional characteristics. To the best of our knowledge, ours is the first study evaluating adult dietary quality by adherence to the WHO dietary recommendations in a nationally representative survey of the Mexican population. We identified those nutrients that are deficient or excessive and studied them according to sociodemographic and nutritional characteristics of the Mexican adult population.

Our indices suggest that Mexico actually presents a double burden of malnutrition - excess and deficiency in some segments of the population and that nutrient policies must be adapted to these nutritional problems.

We observed that participants living in the north of the country complied least with the fruit and vegetable intake recommendation. These findings are in concordance with those of Ramirez et al. (2009) from the same population. These authors reported that persons living in northern Mexico had the lowest intake of fruit and vegetables in the country ${ }^{(31)}$. In our study we observed that rural inhabitants had the highest compliance with recommendations for the CPI, except for the consumption of fruit and vegetables. It has been documented that the urban diet is more diversified and higher in animal products and vegetables as compared with the rural diet ${ }^{(32)}$. In the present study, $<35 \%$ of Mexican adults consumed the recommended intake of fruit and vegetables. Mexico is one of the countries with the lowest consumption of fruit and vegetables worldwide ${ }^{(33)}$.

In our study we observed that participants in the higher SES category barely reached the recommendations for components of the CPI; however, they complied best with the recommended intake of fruit and vegetables, suggesting that their accessibility to fruit and vegetables is better, as other authors have reported ${ }^{(34,35)}$. Our results showed that rural residents, those from southern Mexico and those in the lowest SES tertile consumed a diet with a higher CPI compared with their counterparts: urban residents, those from North, Central and Mexico City, and those from middle and high SES tertiles. Urban residents and participants in the highest SES tertile reported a significantly more diversified and micronutrient-adequate diet than rural residents or participants in the lowest SES tertile. Previous reviews document a strong relationship between dietary diversity and socio-economic characteristics ${ }^{(36-38)}$. Hatloy et al. (2000) documented that socio-economic factors are important determinants for dietary diversity in urban and rural areas ${ }^{(36)}$. It has been reported that dietary diversity increases as income increases ${ }^{(38)}$. Hoddinott and Yohannes (2002) showed that household dietary diversity increases with household food per capita consumption in Bangladesh, Egypt, Ghana, India, Kenya, Malawi, Mali, Mexico, Mozambique and the Philippines ${ }^{(37)}$. 


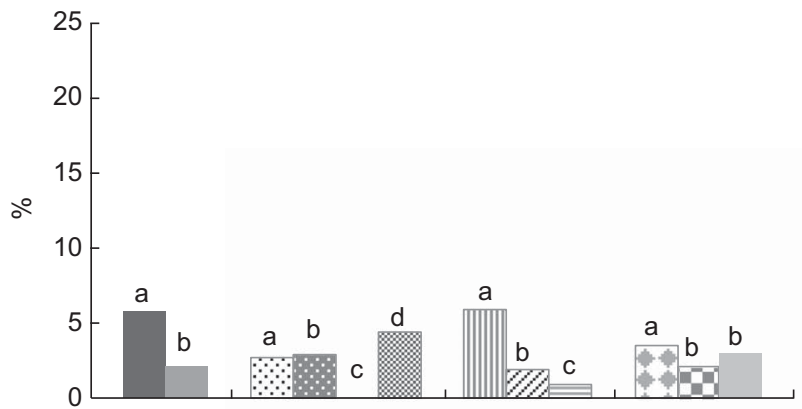

DDI-1: $\leq 7$ different food groups

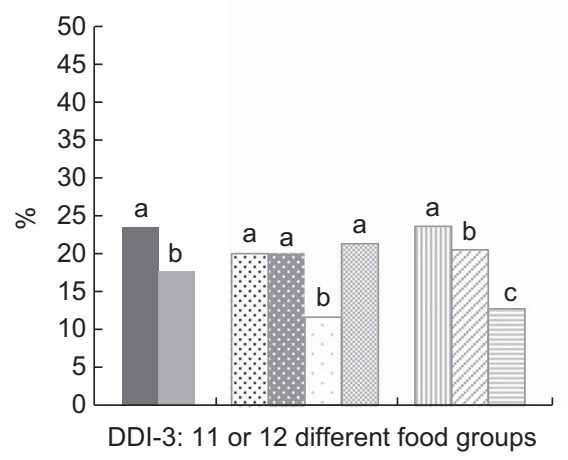

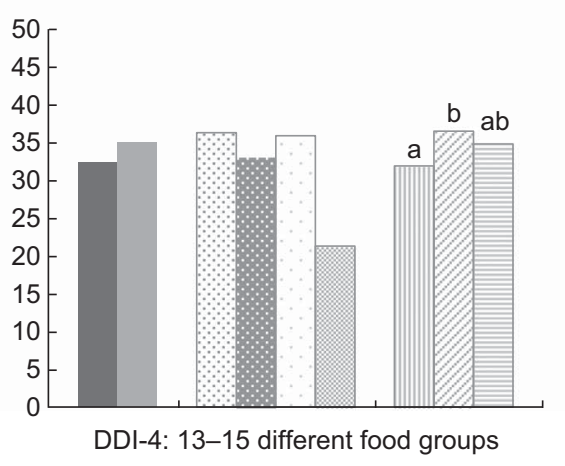

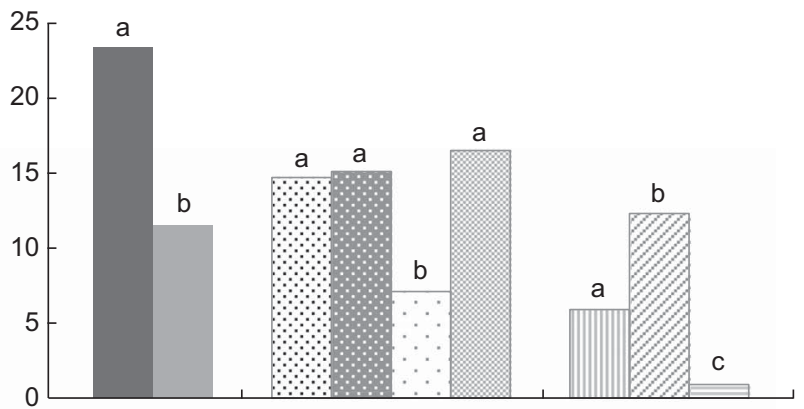

DDI-2: 8-10 different food groups

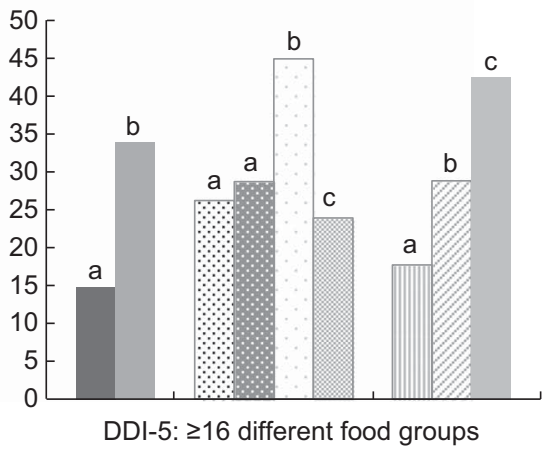

Fig. 3 Daily dietary diversity based on the consumption of thirty different food groups comprising the dietary diversity index (DDI) according to area of residence ( $\square$, rural, $n$ 6452; $\square$, urban, $n$ 9223), geographic region ( $\square$, North, $n$ 2913; $\square$, Centre, $n$ 5929; $\square$, Mexico City, $n$ 667; $\square$, South, $n$ 6166), socio-economic status (SES) tertile (四, low SES, $n$ 7673; $\square$, middle SES, $n$ 5048; 自, high SES, $n$ 2954) and nutritional status (圈, normal weight, $n 5181$; $\square$, overweight, $n 5814 ; \square$, obese, $n$ 4680) among Mexican adults aged 19-59 years, 2006 National Health and Nutrition Survey (ENSANUT-2006). Values are proportions adjusted by age and sex through logistic regression models. ${ }^{a, b, c}$ Adjusted proportions within categories of each independent variable with unlike superscript letters were significantly different $(P<0 \cdot 05)$. The number of food groups consumed for each DDI score (DDI-1 to DDI-5) are are indicated below each plot

In the present study we found that participants from northern Mexico had the lowest CPI. This region is the most industrialized in the country and exposure to a diet rich in fat and total sugar is higher. Our results are consistent with those found by Barquera et al. (2009), who reported that individuals from the north of the country and urban settings had higher intakes of total and saturated fat as well as cholesterol ${ }^{(39)}$. In our study we showed that rural participants presented a more cardioprotective diet compared with urban participants. It has been documented that urban settings lead to an atherogenic diet among Africans ${ }^{(40)}$ and Mexicans ${ }^{(41)}$. A previous study carried out in Mexico reported that urban inhabitants had a higher prevalence of hypercholesterolaemia and hypertriacylglycerolaemia than rural inhabitants ${ }^{(42)}$. The CPI includes nutrients and foods associated with chronic noncommunicable diseases (NCD) such as diabetes mellitus and hypertension, which represent the leading cause of mortality. Thus, it is important to take into account CPI components when establishing policies.

The present study also showed that participants from rural areas and of low SES had the lowest compliance with the recommendations of the MAI. It has been proposed that rural settings have a traditional plant-based diet $^{(43)}$, which is related to a lower risk of developing
CVD $^{(44)}$. Even when our urban participants reported a more diversified diet and, as a consequence, higher micronutrient adequacy, they also presented a diet that stimulates the development of CVD. This confirms our previous results among the Mexican population, where a more diversified diet was significantly associated with a more atherogenic diet ${ }^{(41)}$.

We observed that a significantly higher proportion of overweight or obese participants reached the recommended intakes of fibre and vitamins $\mathrm{A}$ and $\mathrm{C}$ than normal-weight participants. In order to elucidate these results, we compared the intake of these nutrients per $4184 \mathrm{~kJ}$ (1000 kcal) and observed that fibre $(13 \cdot 2 v \cdot 13 \cdot 0 \mathrm{~g} / 4184 \mathrm{~kJ} ; P<0 \cdot 05)$, vitamin A $(299 \cdot 2 v .275 \cdot 7 \mu \mathrm{g} / 4184 \mathrm{~kJ} ; P<0 \cdot 05)$ and vitamin C $(59.8 v .54 .5 \mathrm{mg} / 4184 \mathrm{~kJ} ; P<0.05)$ were significantly higher in overweight and obese than in normal-weight participants (data not shown). Earlier studies have reported that overweight or obese individuals estimate lower intake of energy-dense foods and higher intake of fruit and vegetables; thus, energy intake is under-reported by the overweight or obese ${ }^{(45,46)}$.

Not all indices offer independent information on the dietary consumption of the population or its risk of developing diseases. As expected, dietary diversity and micronutrient adequacy were positively correlated 
( $r=0.435, P<0 \cdot 001$ ), indicating that a diverse diet is accompanied by an increase, albeit partially, in micronutrient density, which is in concordance with other studies $^{(14,47,48)}$. However, the cardioprotective quality of a diet in the adult Mexican population is independent of its nutrient density or diversity. This shows that a Mexican diet may be adequate in one health-related dimension but not in others, and emphasizes the notion that a multidimensional evaluation must be considered when assessing dietary adequacy of a population. The latter has been reported in a previous study ${ }^{(41)}$.

The present study has some limitations. First, the period for data collection does not include nutrient intake from the entire year; however, the period for data collection extended from October 2005 to May 2006 and may have included some of the seasonal availability of fruits and vegetables.

Second, we assessed total Fe consumption without differentiation of haem from non-haem Fe. Bioavailability of non-haem Fe depends on the presence of other dietary components consumed during the same day. We cannot estimate the bioavailability of intake of dietary Fe from our population with the available data. Furthermore, there are no specific dietary recommendations for haem $\mathrm{Fe}$. Thus, total Fe consumption is an ambiguous indicator of Fe intake that may under- or overestimate adequacy of true dietary Fe availability.

Because the study was cross-sectional and observational, causality cannot be determined. However, because of the representative sample of the Mexican population we can identify those recommendations that should be improved in dietary guidelines.

We did not assess $\mathrm{Na}$ intake because the FFQ is not an adequate method for its estimation ${ }^{(49)}$; the same for free sugars. The components $n-3$ and $n-6$ fatty acids, transfatty acids and free sugars were not included in the CPI due to lack of information on local food composition at the time of data analysis. There is no specific recommendation for MUFA; thus, this was not measured. Therefore we did not include all WHO dietary recommendations for the prevention of CVD. Dietary diversity is defined as the number of different food groups consumed during a reference period ${ }^{(19)}$. However, our DDS does not allow us to distinguish healthy food groups such as whole grains from unhealthy food groups such as highly energy-dense foods. Therefore, we may be underestimating the at-risk population.

The lack of validation of our indices is also a shortcoming of our study; however, as we previously noted, the objective of our study was to observe the adult Mexican diet from the standpoint of dietary quality. We are aware that the ideal dietary index is that which is developed in relation to nutritional profiles or health indicators. Otherwise, its validity to infer dietary risk or protection is compromised.

However, there is a vast body of literature showing that some dietary characteristics offer unequivocal risk or protection against certain health conditions. Such is the case for salt in relation to hypertension, saturated or trans-fatty acids in relation to heart disease and atherosclerosis, fibre in relation to colon cancer, and $\mathrm{Fe}$ in relation to anaemia. These are all significant diseases in Mexico. We analysed the national diet of Mexican adults by applying solid scientific evidence of causation and not with the intention of exploring new dietary characteristics. Based on well-known dietary risk factors or protectors, our intention was to characterize and describe the major features and to elucidate that dietary analysis is a complex multidimensional concept. We are adapting solid research into high-quality guidelines for local use as an efficient way to improve the applicability of evidenceinformed dietary recommendations.

The strength of the present study is that we draw inference on the diet of the complete adult Mexican population and can offer insights into its strengths and weaknesses, as well as individualized recommendations with national representation.

Validation of indices is necessary and is our next step; however, components of the indices used have been validated in other studies. For instance, the Healthy Diet Indicator was developed according to WHO guidelines for the prevention of chronic diseases and was shown to be inversely associated with all-cause mortality in males from Finland, Italy and the Netherlands ${ }^{(50)}$. The Diet Quality Index Revised developed by Hains et al. ${ }^{(51)}$ was reported to correlate with plasma biomarkers ${ }^{(52)}$ for micronutrient intake. Dietary variety and diversity were shown to be associated with nutrient adequacy ${ }^{(53)}$, biomarkers ${ }^{(54)}$ and lower disease risk ${ }^{(55)}$.

These indices, with some modifications, have been used previously in Mexicans ${ }^{(41)}$, Haitian immigrants in Canada $^{(56)}$ and a sample of the African population ${ }^{(40)}$, but not in a nationally representative sample.

The components of our indices provided qualitative information related to problems of under- and overnutrition. Our indices allow recognition of both under- and overnutrition and identify groups with poor dietary quality as well as characterize different types of poor dietary quality. These indices also allow characterization of nutritional problems such as micronutrient deficiencies or excess of particularly high-risk food components, both of which are present in populations undergoing nutritional transition. These indices may be used for monitoring dietary quality according to different sociodemographic and nutritional characteristics of the Mexican population. We did not use indices used in other studies such as the Healthy Eating Index ${ }^{(57)}$ or the Diet Quality Index developed by Patterson et al. ${ }^{(58)}$ because we consider that they may not be useful for the Mexican population, being designed for populations with different characteristics. Further research may be aimed at validating these indices with nutritional biomarkers or health outcomes. Due to the mixture of dietary 
characteristics from the diverse populations living in countries undergoing nutritional transition, it may be inappropriate to evaluate only one dietary feature. Our indices allowed measurement of the overall dietary quality, micronutrient density, dietary diversity and cardioprotective effects, avoiding problems of a specific nutrient or foods related to diseases.

However, even though the three dietary indices identified dietary inadequacies, due to the alarming increase in obesity and NCD in Mexico ${ }^{(1)}$, we believe that among the three dietary indices reviewed, the CPI from adult diets is the one that may be useful to characterize the diet and to monitor programmed recommendations. Thus, validation of the CPI with nutritional biomarkers or health outcomes is relevant. However, nutritional policies aimed to prevent chronic NCD have been created from the knowledge and evidence of different interventions. For example, the Mexican National Agreement for Dietary Health $^{(59)}$, the $\mathrm{WHO}^{(16)}$ and the US Institute of Medicine ${ }^{(60)}$ promote reductions in $\mathrm{Na}$, sugar and fat intakes as a means to improve health and to decrease incidence of chronic diet-related NCD.

\section{Conclusion}

Our findings offer evidence that there is not a sufficiently adequate single index to identify all relevant characteristics of a healthy diet (such as micronutrient adequacy, cardioprotective diet or dietary diversity). There are several approaches to evaluate diets, all related to health in a meaningful way. We have offered evidence that it is insufficient to evaluate only one characteristic of the diet: its micronutrient density, diversity, or even its cardioprotective characteristics. We have shown that a diverse and micronutrient-rich diet may not necessarily be a healthy diet because it may also be atherogenic. Likewise, a cardioprotective diet may be poor in micronutrients. We suggest that these three dimensions be considered in dietary evaluation with a special focus on the CPI. Hence, in countries such as Mexico that are undergoing the nutrition transition, dietary guidelines should include recommendations on improving diversity and the micronutrient density of diets. Above all, guidelines should focus on reducing fat, $\mathrm{Na}$ and sugars. These nutrients are associated with chronic NCD such as diabetes mellitus and hypertension, which represent the leading cause of mortality. In addition, the energy density of the diet, fibre content, amount of processed foods and other aspects should also be investigated and evaluated to develop healthier recommendations for the population.

\section{Acknowledgements}

Sources of funding: Funding for this research was provided by the NIPH. Analysis of the data, interpretation of the findings and preparation of the manuscript were conducted in the NIPH. The NIPH had no role in the design, analysis or writing of this article. Conflicts of interest: The authors declare that they have no competing interests; however, they have had received funds from the food-related industry for projects different from the present analysis. T.G.d.C. participates in the DANONE research grant which evaluates the impact of promoting water consumption by obese young women, but does not receive funding from this project. She is also a part of a scientific group which reviews annual work plans for the Kelloggs' Nutrition and Health Institute in Mexico, for which she also evaluates research proposals submitted for funding. She received \$35000 Mexican pesos (roughly \$US 2700) in 2012 for these annual activities. S.R.-R. participated in the DANONE research grant which evaluates the impact of promoting water consumption by obese young women. S.B. participated in two NIPH water consumption studies sponsored by DANONE and is part of the Hydration for Health Scientific Group. He has received honoraria (under \$US 4000) from AstraZeneca, Herbalife and Medix to participate in scientific or medical meetings describing the epidemiology of obesity and NCD in Mexico in the last 4 years. T.S. received funding from Nestlé in 2012 to validate by triplicate a $24 \mathrm{~h}$ recall questionnaire in a national sample in Mexico (\$US 1000000 ). In 2013, she directed two nutritional status assessment workshops for paediatricians; the payment for each one of them was \$US 1000. V.M.-R. and X.P. have not received funding from the food-related industry. Authors' contributions: T.G.d.C. was the lead author; X.P. was responsible for formulating the research questions and writing the draft; X.P. and S.R.-R carried out the statistical analysis; V.M.-R. S.R.-R., T.S., T.G.d.C. and S.B. worked in close collaboration with the first author analysing the data and interpreting the results; all authors provided critical revision and approved the final manuscript.

\section{References}

1. Rivera JA, Barquera S, Campirano F et al. (2002) Epidemiological and nutritional transition in Mexico: rapid increase of non-communicable chronic diseases and obesity. Public Health Nutr 5, 113-122.

2. Rojas R, Aguilar-Salinas C, Jiménez-Corona A et al. (2010) Metabolic syndrome in Mexican adults: results from the National Health and Nutrition Survey 2006. Salud Publica Mex 52, Suppl. 1, S11-S18.

3. Olaiz G, Rivera JA \& Shamah T (2006) Encuesta Nacional de Nutrición y Salud 2006 (ENSANUT-2006). Cuernavaca, Morelos: Instituto Nacional de Salud Pública.

4. Rivera JA \& Sepulveda Amor J (2003) Conclusions from the Mexican National Nutrition Survey 1999: translating results into nutrition policy. Salud Publica Mex 45, Suppl. 4, S565-S575.

5. Barquera S, Campos I, Hernández-Barrera L et al. (2009) Obesity and central adiposity in Mexican adults: results from the Mexican National Health and Nutrition Survey 2006. Salud Publica Mex 51, Suppl. 4, S595-S603. 
6. Barquera S, Peterson KE, Must A et al. (2007) Coexistence of maternal central adiposity and child stunting in Mexico. Int J Obes (Lond) 31, 540-550.

7. Lifshitz F (2009) Nutrition and growth. J Clin Res Pediatr Endocrinol 14, 157-163.

8. Kant AK (2010) Dietary patterns: biomarkers and chronic disease risk. Appl Physiol Nutr Metab 35, 199-206.

9. Rizzo NS, Sabaté J, Jaceldo-Siegl K et al. (2011) Vegetarian dietary patterns are associated with a lower risk of metabolic syndrome: the Adventist Health Study 2. Diabetes Care 34 , 1225-1227.

10. World Health Organization \& Food and Agriculture Organization of the United Nations (1996) Preparation and Use of Food Based Dietary Guidelines. Report of a Joint FAO/WHO Consultation, Nicosia, Cyprus. Geneva: WHO.

11. Waijers PM, Feskens EJ \& Ocké MC (2007) A critical review of predefined diet quality scores. BrJ Nutr 97, 219-231.

12. Fransen HP \& Ocké MC (2008) Indices of diet quality. Curr Opin Clin Nutr Metab Care 11, 559-565.

13. Krebs-Smith SM \& Clark LD (1989) Validation of a nutrient adequacy score for use with women and children. $J \mathrm{Am}$ Diet Assoc 89, 775-783.

14. Kennedy GL, Pedro MR, Seghieri C et al. (2007) Dietary Diversity Score is a useful indicator of micronutrient intake in non-breast-feeding Filipino children. J Nutr 137, 472-477.

15. Kennedy ET, Ohls J, Carlson S et al. (1995) The Healthy Eating Index: design and applications. J Am Diet Assoc 95, 1103-1108

16. World Health Organization \& Food and Agriculture Organization of the United Nations (2003) Diet, Nutrition and Prevention of Chronic Diseases. Report of a Joint WHO/FAO Expert Consultation. WHO Technical Report Series no. 916. Geneva: WHO.

17. Mazzocchi M, Brasili C \& Sandri E (2007) Trends in dietary patterns and compliance with World Health Organization recommendations: a cross-country analysis. Public Health Nutr 11, 535-540.

18. Foote JA, Murphy SP, Wilkens LR et al. (2004) Dietary variety increases the probability of nutrient adequacy among adults. J Nutr 134, 1779-1785.

19. Ruel MT (2002) Is Dietary Diversity an Indicator of Food Security or Dietary Quality? A Review of Measurement Issues and Research Needs. Discussion Paper no. 140. Washington, DC: International Food Policy Research Institute.

20. Adda J, Chandola T \& Michael M (2003) Socio-economic status and health: causality and pathways. J Econom 112 , 57-63.

21. Wirt A \& Collins CE (2009) Diet quality - what is it and does it matter? Public Health Nutr 12, 2473-2492.

22. Rodríguez-Ramírez S, Mundo-Rosas V, Jiménez-Aguilar A et al. (2009) Methodology for the analysis of dietary data from the Mexican National Health and Nutrition Survey 2006. Salud Publica Mex 51, Suppl. 4, S523-S529.

23. Safdie M, Barquera S, Porcayo M et al. (2004) Bases de datos de valor nutritivo de los alimentos. Compilación del Instituto Nacional de Salud Pública. Cuernavaca, Morelos: Instituto Nacional de Salud Pública.

24. Lohman T, Roche A \& Martorell R (1988) Anthropometric Standarization Reference Manual. Champaign, IL: Human Kinetics.

25. Habicht JP (1974) Estandarización de métodos epidemiológicos cuantitativos sobre el terreno (Standardization of anthropometric methods in the field). PAHO Bull $\mathbf{7 6}$, 375-384.

26. World Health Organization (2000) Obesity: Preventing and Managing the Global Epidemic. Report on a WHO Consultation. WHO Technical Report Series no. 894. Geneva: WHO.

27. Resano-Pérez E, Méndez-Ramírez I, Shamah-Levy T et al. (2003) Methods of the National Nutrition Survey 1999. Salud Publica Mex 45, Suppl. 4, S558-S564.
28. Institute of Medicine, Food and Nutrition Board (2000) Dietary Reference Intakes for Vitamin C, Vitamin E, Selenium and Caratenoids. Washington, DC: National Academy Press.

29. Institute of Medicine, Food and Nutrition Board (1997) Dietary Reference Intakes for Calcium, Phosphorus, Magnesium, Vitamin D, and Fluoride. Washington, DC: National Academy Press.

30. Institute of Medicine, Food and Nutrition Board (1998) Dietary References Intakes for Thiamin, Riboflavin, Niacin, Vitamin $B_{6}$, Folate, Vitamin $B_{12}$, Pantothenic Acid, Biotin and Cholin. Washington, DC: National Academy Press.

31. Ramírez-Silva I, Rivera JA, Ponce X et al. (2009) Fruit and vegetable intake in the Mexican population: results from the Mexican National Health and Nutrition Survey 2006. Salud Publica Mex 51, Suppl. 4, S574-S585.

32. Delisle H (1990) Patterns of Urban Food Consumption in Developing Countries: Perspective from the 1980's. Rome: FAO.

33. Lock K, Pomerleau J, Causer L et al. (2005) The global burden of disease attributable to low consumption of fruit and vegetables: impliation for the global strategy on diet. Bull World Health Organ 83, 100-108.

34. Subar AF, Heimendinger J, Patterson BH et al. (1995) Fruit and vegetable intake in the United States: the baseline survey of the Five A Day for Better Health Program. Am J Health Promot 9, 352-360.

35. Ball K, Crawford D \& Mishra G (2006) Socio-economic inequalities in women's fruit and vegetable intakes: a multilevel study of individual, social and environmental mediators. Public Health Nutr 9, 623-630.

36. Hatloy A, Hallund J, Diarra MM et al. (2000) Food variety, socioeconomic status and nutritional status in urban and rural areas in Koutiala (Mali). Public Health Nutr 3, 57-65.

37. Hoddinot J \& Yohannes Y (2002) Dietary Diversity as Housebold Food Security Indicator. Discussion Paper no. 136. Washington, DC: International Food Policy Research Institute.

38. Ruel MT (2003) Operationalizing dietary diversity: a review of measurement issues and research priorities. J Nutr 133, 11 Suppl. 2, 3911S-3926S

39. Barquera S, Hernández-Barrera L, Campos-Nonato I et al. (2009) Energy and nutrient consumption in adults: analysis of the Mexican National Health and Nutrition Survey 2006. Salud Publica Mex 51, Suppl. 4, S562-S573.

40. Sodjinou R, Agueh V, Fayomi B et al. (2007) Dietary patterns of urban adults in Benin: relationship with overall diet quality and socio-demographic characteristics. Eur $J$ Clin Nutr 63, 222-228.

41. Ponce X, Ramirez E \& Delisle H (2006) A more diversified diet among Mexican men may be also more atherogenic. J Nutr 136, 2921-2927.

42. Aguilar-Salinas C, Gómez-Pérez F, Rull J et al. (2010) Prevalence of dyslipidemias from the National Health and Nutrition Survey 2006. Salud Publica Mex 52, Suppl. 1, S44-S53.

43. Solomons NW (2000) Plant based diets are traditional in developing countries: 21st century challenges for better nutrition and health. Asia Pac J Clin Nutr 9, Suppl, S41-S54.

44. Hu FB (2003) Plant-based foods and prevention of cardiovascular disease: an overview. Am J Clin Nutr 78, 3 Suppl., 544S-551S.

45. Mendez MA, Wynter S, Wilks R et al. (2004) Under- and overreporting of energy is related to obesity, lifestyle factors and food group intakes in Jamaican adults. Public Health Nutr 7, 9-19.

46. Millen AE, Tooze JA, Subar AF et al. (2009) Differences between food group reports of low-energy reporters and non-low-energy reporters on a food frequency questionnaire. J Am Diet Assoc 109, 1194-1203. 
47. Arsenault JE, Yakes EA, Islam MM et al. (2013) Very low adequacy of micronutrient intakes by young children and women in rural Bangladesh is primarily explained by low food intake and limited diversity. J Nutr 143, 197-203.

48. Arimond M, Wiesmann D, Becquey E et al. (2010) Simple food group diversity indicators predict micronutrient adequacy of women's diets in 5 diverse, resource-poor settings. J Nutr 140, issue 11, 259S-269S.

49. Sarno F (2009) Estimated sodium intake by the Brazilian population, 2002-2003. Rev Saude Publica 43, 1-6.

50. Huijbregts P, Feskens E, Rasanen L et al. (1997) Dietary pattern and 20 year mortality in elderly men in Finland, Italy, and The Netherlands: longitudinal cohort study. BMJ 315, 13-17.

51. Haines PS, Siega-Riz AM \& Popkin BM (1999) The Diet Quality Index revised: a measurement instrument for populations. J Am Diet Assoc 99, 697-704.

52. Newby PK, Hu FB, Rimm EB et al. (2003) Reproducibility and validity of the Diet Quality Index Revised as assessed by use of a food-frequency questionnaire. Am J Clin Nutr 78, 941-949.

53. Bernstein MA, Tucker KL, Ryan ND et al. (2002) Higher dietary variety is associated with better nutritional status in frail elderly people. J Am Diet Assoc 102, 1096-1104.
54. Vyncke K, Cruz Fernandez E, Fajó-Pascual M et al. (2013) Validation of the Diet Quality Index for Adolescents by comparison with biomarkers, nutrient and food intakes: the HELENA study. BrJ Nutr 109, 2067-2078.

55. Fernandez E, Negri E, La Vecchia C et al. (2000) Diet diversity and colorectal cancer. Prev Med 31, 11-14.

56. Désilets MC, Rivard M, Shatenstein B et al. (2007) Dietary transition stages based on eating patterns and diet quality among Haitians of Montreal, Canada. Public Health Nutr 10, 454-463.

57. Basiotis PP, Carlson A, Gerrior SA et al. (2002) The Healthy Eating Index: 1999-2000. Washington, DC: US Departement of Agriculture, Center for Nutrition Policy and Promotion.

58. Patterson RE, Haines PS \& Popkin BM (1994) Diet quality index: capturing a multidimensional behavior. J Am Diet Assoc 94, 57-64.

59. Secretaría de Salud (2010) Acuerdo Nacional para la Salud Alimentaria. Estrategia contra el Sobrepeso y la Obesidad. México, DF: Secretaría de Salud.

60. Committee on an Evidence Framework for Obesity Prevention Decision Making, Institute of Medicine (2010) Bridging the Evidence Gap in Obesity Prevention: A Framework to Inform Decision Making [SK Kumanyika, L Parker and LJ Sim, editors]. Washington, DC: The National Academies Press. 